\title{
BER analysis of TDD downlink multiuser MIMO systems with imperfect channel state information
}

\author{
Baolong Zhou ${ }^{1,2^{*}}$, Lingge Jiang ${ }^{1}$, Shengjie Zhao ${ }^{2}$ and Chen $\mathrm{He}^{1}$
}

\begin{abstract}
In downlink multiuser multiple-input multiple-output (MU-MIMO) systems, the zero-forcing (ZF) transmission is a simple and effective technique for separating users and data streams of each user at the transmitter side, but its performance depends greatly on the accuracy of the available channel state information (CSI) at the transmitter side. In time division duplex (TDD) systems, the base station estimates CSI based on uplink pilots and then uses it through channel reciprocity to generate the precoding matrix in the downlink transmission. Because of the constraints of the TDD frame structure and the uplink pilot overhead, there inevitably exists CSI delay and channel estimation error between CSI estimation and downlink transmission channel, which degrades system performance significantly. In this article, by characterizing CSI inaccuracies caused by CSI delay and channel estimation error, we develop a novel bit error rate (BER) expression for M-QAM signal in TDD downlink MU-MIMO systems. We find that channel estimation error causes array gain loss while CSI delay causes diversity gain loss. Moreover, CSI delay causes more performance degradation than channel estimation error at high signal-to-noise ratio for time varying channel. Our research is especially valuable for the design of the adaptive modulation and coding scheme as well as the optimization of MU-MIMO systems. Numerical simulations show accurate agreement with the proposed analytical expressions.
\end{abstract}

Keywords: BER, channel estimation error, delay, MU-MIMO, TDD, zero forcing

\section{Introduction}

Owing to their high spectral efficiency, multiple-input multiple-output (MIMO) wireless antenna systems have been recognized as a key technology for future wireless communication systems such as long-term evolution (LTE), LTE-advanced (LTE-A), WiMax, etc. Multiuser MIMO (MU-MIMO) has become one of the main features in LTE-A systems because of several key advantages over single-user MIMO (SU-MIMO) [1,2]. There are several kinds of classic transmission methods for downlink MU-MIMO: Dirty Paper Coding (DPC) [3], Block Diagonalization (BD) $[4,5]$, and zero-forcing (ZF) (or channel inversion) [6]. Though DPC is optimal and can achieve sum-rate capacity, it is difficult to implement in practical systems because of its high complexity. $\mathrm{BD}$ and ZF are suboptimal methods with tolerable performance degradation and their lower complexities

\footnotetext{
* Correspondence: baolong.zhou@alcatel-sbell.com.cn

'Department of Electronic Engineering, Shanghai Jiao Tong University, Shanghai, P.R. China

Full list of author information is available at the end of the article
}

make them easier to implement. Furthermore, compared to $\mathrm{BD}, \mathrm{ZF}$ is an even simpler algorithm which essentially separates multiple data streams from the same user equipment (UE) at transmitter side. So, in this article, ZF transmission method is chosen for the performance analysis of downlink MU-MIMO systems; however, similar analytical methods may be applied to other transmission methods as well.

As is well known, the availability of accurate channel state information (CSI) is very important for downlink MU-MIMO schemes. However, in practice, CSI is always imperfect because of the existence of CSI delay, quantization error, and channel estimation error. This would cause not only self-interference among different data streams of the same user, but also interference among users, severely degrading the performance especially in case of high mobile users or long delay. Hence, it is important to characterize the performance of MUMIMO system in the presence of imperfect CSI.

Most recent study [7-12] about the impact of imperfect CSI on MU-MIMO focused on the frequency 
division duplex systems. In [7], the authors investigated the impact of feedback delay and estimation error on the sum-rate of MU-MIMO systems. In [8], the authors studied upper and lower bounds on the achievable sumrate of a correlated/uncorrelated MU-MIMO channel with channel estimation error and feedback delay. The achievable ergodic rates were derived for multi-user MIMO systems with CSI delay and quantization error in $[9,10]$. In [11], the impact of imperfect CSI on sumrate scaling law was investigated for downlink MUMIMO systems. In [12], the authors quantified the impact of channel estimation errors, quantization errors, and outdated quantized CSI on the rate loss of MUMIMO system.

To the authors' knowledge, the impact of imperfect CSI on MU-MIMO in time division duplex (TDD) systems is almost rarely investigated. In this article, we study the impact of imperfect CSI caused by both CSI delay and channel estimation error on bit error rate (BER) for TDD downlink MU-MIMO ZF systems. In order to clearly indicate the impact of imperfect CSI on MU-MIMO, we only analyze un-coded MU-MIMO systems although channel coding techniques are indispensable in practical systems. In TDD system, the base station (BS) estimates CSI at transmitter side based on the uplink pilots periodically sent by the mobile users. Then, BS uses it through channel reciprocity to generate precoding matrix for the downlink data transmission. Because of the constraints of the TDD frame structure and the uplink pilot overhead, there inevitably exists both CSI delay and channel estimation error between uplink estimated channel (used to generate precoding matrix during downlink transmission) and downlink transmission channel, which degrades the system performance. In this article, using the correlation between the actual channel and the estimated one [13], as well as the channel's time-correlation [14], we obtain an expression for post-processing signal-tointerference plus noise ratio (SINR) of each data stream of TDD downlink MU-MIMO systems. Based on the post-processing SINR, we then obtain the expression for average BER of uncoded TDD MUMIMO ZF systems with M-quadrature amplitude modulation (QAM)-modulated signals. Numerical simulations verify our analysis.

Notation: $E(\cdot),(\cdot)^{\mathrm{H}},(\cdot)^{\mathrm{T}},(\cdot)^{*}$, and $\|\cdot\|_{\mathrm{F}}$ denote expectation, Hermitian, transpose, complex conjugation, and Frobenius norm, respectively. $\mathbf{I}_{M}$ is the $M \times M$ identity matrix. $(\cdot)^{\dagger}$ denotes the right pseudo inversion and $(\mathrm{A})^{\dagger} \triangleq \mathrm{A}^{\mathrm{H}}\left(\mathrm{AA}^{\mathrm{H}}\right)^{-1} \cdot \mathcal{C N}(\boldsymbol{\mu}, \boldsymbol{\Sigma})$ denotes the complex Gaussian distribution with mean vector $\boldsymbol{\mu}$ and variance matrix $\boldsymbol{\Sigma}$.

\section{System model}

Consider a TDD downlink MU-MIMO system with ZF precoding, where a BS equipped with $M$ antennas transmits signals to $K$ mobile users, each equipped with $n_{k}$ ( $k$ $=1,2, \ldots, K)$ antennas, under the assumption that $M \geq N=\sum_{k=1}^{K} n_{k}$ to guarantee the existence of a nonzero precoding matrix. This assumption can be satisfied with the help of user scheduling techniques which select a subset (active users) of the available users to communicate at each time slot such that the total number of receive antennas for active users at any time instant satisfies the above required assumption $[15,16]$. Because orthogonal frequency division multiplexing divides a wideband MIMO channel into a series of parallel narrowband MIMO channels, we can assume that the channels are frequency flat. Furthermore, we assume that the channels are spatially uncorrelated, time-varying, and Rayleigh fading, and channel's power spectrum follows Jakes model [17]. The channel matrix from the BS to the $k$ th user is denoted by $\mathbf{H}_{k}=\left\{h_{k, i j}\right\}_{n_{k} \times M^{\prime}}$ where $h_{k, i j} \sim \mathcal{C N}(0,1)$ is the complex channel gain between the $j$ th transmit antenna of $\mathrm{BS}$ and the $i$ th receive antenna of user $k$. Let $\mathbf{b}_{k}$ denote the $n_{k} \times 1$ transmit signal vector to user $k$. This signal vector is first multiplied by an $M \times n_{k}$ precoding matrix $\mathbf{T}_{k}$ and then transmitted through $M$ transmit antennas. The received signal vector $\mathbf{y}_{k}\left(n_{k} \times 1\right)$ of user $k$ at the $m$ th symbol interval is

$$
\mathbf{y}_{k}[m]=\mathbf{H}_{k}[m] \sum_{l=1}^{K} \mathbf{T}_{l}[m] \mathbf{b}_{l}[m]+\mathbf{n}_{k}[m],
$$

where $\mathbf{n}_{k} \sim \mathcal{C N}\left(0, N_{0, k} \mathbf{I}_{n_{k}}\right)$ is an additive white Gaussian noise (AWGN) vector, $\mathbf{b}_{l}$ satisfies $\mathrm{E}\left[\mathbf{b}_{l} \mathbf{b}_{l}^{\mathrm{H}}\right]=E_{\mathrm{s}} \mathbf{I}_{n_{l}}$, $E_{\mathrm{s}}$ is the symbol energy. The system equation can be expressed in the matrix form as follows

$$
\mathbf{y}[m]=\mathbf{H}[m] \mathbf{T}[m] \mathbf{b}[m]+\mathbf{n}[m],
$$

where

$$
\begin{aligned}
& \mathbf{y}[m] \triangleq\left[\mathbf{y}_{1}^{\mathrm{T}}[m] \mathbf{y}_{2}^{\mathrm{T}}[m] \ldots \mathbf{y}_{K}^{\mathrm{T}}[m]\right]^{\mathrm{T}}, \quad \mathbf{H} \triangleq\left[\mathbf{H}_{1}^{\mathrm{T}}[m] \mathbf{H}_{2}^{\mathrm{T}}[m] \ldots \mathbf{H}_{K}^{\mathrm{T}}[m]\right]^{\mathrm{T}}, \quad \mathrm{T}[m] \triangleq\left[\mathbf{T}_{1}[m] \mathbf{T}_{2}[m] \ldots \mathbf{T}_{K}[m]\right], \\
& \mathbf{b}[m] \triangleq\left[\mathbf{b}_{1}^{\mathrm{T}}[m] \mathbf{b}_{2}^{\mathrm{T}}[m] \ldots \mathbf{b}_{\kappa}^{\mathrm{T}}[m]\right]^{\mathrm{T}}, \quad \mathbf{n}[m] \triangleq\left[\mathbf{n}_{1}^{\mathrm{T}}[m] \mathbf{n}_{2}^{\mathrm{T}}[m] \ldots \mathbf{n}_{K}^{\mathrm{T}}[m]\right]^{\mathrm{T}} .
\end{aligned}
$$

There are seven kinds of TDD frame configurations as defined in 3GPP specifications $[18,19]$. Without loss of generality, we choose the TDD frame configuration 2 for analysis. Figure 1 describes the structure of TDD frame configuration 2 . One radio frame includes $10 \mathrm{sub}-$ frames and 1 subframe (duration is $1 \mathrm{~ms}$ ) includes 14 symbols per subcarrier. The uplink pilots for downlink beamforming transmission can be sent via the last one or several symbols in the special subframe and/or the uplink subframe. 


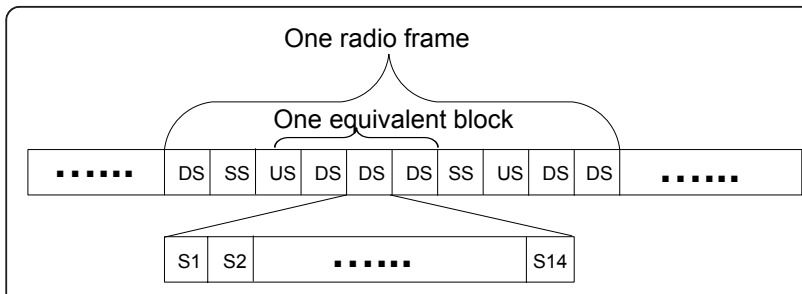

DS: downlink subframe ; US: uplink subframe

SS: special subframe; $\quad S n$ : the $n$-th symbol, $n=1,2, \ldots 14$

Figure 1 TDD frame configuration 2 .

Because only frequency flat fading channel is considered, Figure 1 can be equivalently simplified into Figure 2 for our analysis. Here, all pilot symbols are drawn in one equivalent block together with those data symbols in the sequent downlink subframes, which clearly indicates the CSI delay (denoted by $M_{\mathrm{d}}$ ) between uplink channel estimation and downlink data transmission. In practical TDD systems, the number of pilot symbols (denoted by $n_{\mathrm{p}}$ ) in one equivalent block is very small compared with that of data symbols (denoted by $n_{\mathrm{d}}$ ), so we can consider that all pilot symbols in one equivalent block experience the stationary channel.

In TDD MU-MIMO systems, the procedures at the physical layer for the downlink data transmission are as follows:

Step 1: BS obtains the delay estimated version $\hat{\mathbf{H}}\left[m-M_{\mathrm{d}}\right]$ of CSI based on the received uplink pilots at the $\left(m-M_{\mathrm{d}}\right)$ th symbol interval. Here, $\hat{\mathbf{H}}\left[m-M_{\mathrm{d}}\right] \triangleq\left[\hat{\mathbf{H}}_{1}^{\mathrm{T}}\left[m-M_{\mathrm{d}}\right] \hat{\mathbf{H}}_{2}^{\mathrm{T}}\left[m-M_{\mathrm{d}}\right], \ldots, \hat{\mathbf{H}}_{K}^{\mathrm{T}}\left[m-M_{\mathrm{d}}\right]\right]$, $M_{\mathrm{d}}$ denotes the delay in symbol between the uplink channel estimation and downlink data transmission, and the value of $M_{\mathrm{d}}$ ranges from 1 to $n_{\mathrm{d}}$ for the different downlink data symbol as in Figure 2.

Step 2: BS generates the normalized ZF precoding matrix as follows and sends out the downlink data streams.

$$
\mathbf{T}[m]=\frac{\left(\hat{\mathbf{H}}\left[m-M_{\mathrm{d}}\right]\right)^{\dagger}}{\left\|\left(\hat{\mathbf{H}}\left[m-M_{\mathrm{d}}\right]\right)^{\dagger}\right\|_{\mathrm{F}}}
$$

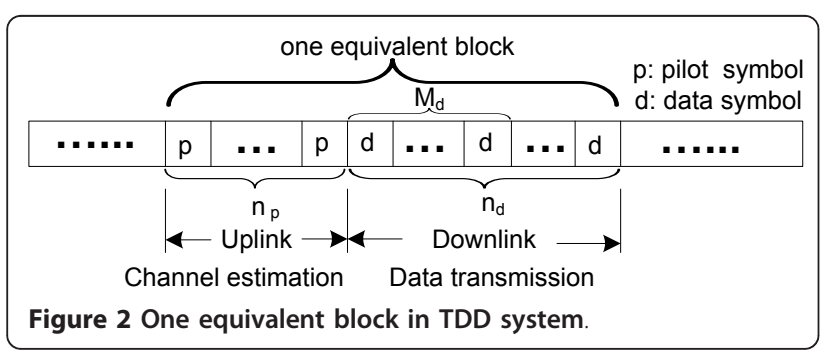

Step 3: each user estimates the downlink channel through the downlink pilots and then detects the received signal.

\section{BER analysis}

In this section, we first derive the post-processing SINR under the given $\hat{\mathbf{H}}\left[m-M_{\mathrm{d}}\right]$, and then derive the average BER based on post-processing SINR.

Substituting (3) into (2), the received signal vector (2) of system can be expressed as

$$
\mathbf{y}[m]=\mathbf{H}[m] \frac{\left(\hat{\mathbf{H}}\left[m-M_{\mathbf{d}}\right]\right)^{\dagger}}{\left\|\left(\hat{\mathbf{H}}\left[m-M_{\mathbf{d}}\right]\right)^{\dagger}\right\|_{\mathbf{F}}} \mathbf{b}[m]+\mathbf{n}[m] .
$$

Similar to [13], we can deduce that $\mathbf{H}\left[m-M_{\mathrm{d}}\right]$ and $\hat{\mathbf{H}}\left[m-M_{\mathrm{d}}\right]$ are jointly complex Gaussian distributed

$$
\left[\begin{array}{l}
\mathbf{H}_{1}\left[m-M_{\mathrm{d}}\right] \\
\mathbf{H}_{2}\left[m-M_{\mathrm{d}}\right] \\
\cdots \\
\mathbf{H}_{K}\left[m-M_{\mathrm{d}}\right]
\end{array}\right]=\left[\begin{array}{l}
\rho_{e, 1} \hat{\mathbf{H}}_{1}\left[m-M_{\mathrm{d}}\right] \\
\rho_{\mathrm{e}, 2} \hat{\mathbf{H}}_{2}\left[m-M_{\mathrm{d}}\right] \\
\cdots \\
\rho_{\mathrm{e}, K} \hat{\mathbf{H}}_{K}\left[m-M_{\mathrm{d}}\right]
\end{array}\right]+\left[\begin{array}{l}
\sqrt{1-\left|\rho_{\mathrm{e}, 1}\right|^{2}} \zeta_{1}\left[m-M_{\mathrm{d}}\right] \\
\sqrt{1-\left|\rho_{\mathrm{e}, 2}\right|^{2}} \zeta_{2}\left[m-M_{\mathrm{d}}\right] \\
\cdots \\
\sqrt{1-\left|\rho_{e, K}\right|^{2}} \zeta_{K}\left[m-M_{\mathrm{d}}\right]
\end{array}\right],
$$

where the elements of $N \times M$ random matrix $\hat{\mathbf{H}}\left[m-M_{\mathrm{d}}\right]$ are independent and identically distributed (i.i.d) zero-mean complex Gaussian random variables with unit variance, the elements of the $n_{k} \times M$ random matrix $\zeta_{k}\left[m-M_{\mathrm{d}}\right]$ are also i.i.d zero-mean complex Gaussian random variables with unit variance, $\rho_{\mathrm{e}, k}$ is the complex correlation coefficient between the actual channel gain and its estimation for user $k$ and is defined as

$$
\rho_{e, k} \triangleq \mathrm{E}\left\{h_{k, i j}\left[m-M_{\mathrm{d}}\left|\hat{h}_{k, j}^{*}\right| m-M_{\mathrm{d}}\right]\right\}, \quad k=1,2, \ldots, K, \quad i=1,2, \ldots, n_{k,}, \quad j=1,2, \ldots, \mathrm{M},
$$

where $0 \leq\left|\rho_{\mathrm{e}, k}\right| \leq 1$. Because the SNR of pilots of each user can be different, $\rho_{\mathrm{e}, k}$ of each user can be different.

Assuming channel follows a Gauss-Markov autoregressive (AR) process, similar to [14] we can also deduce that $\mathbf{H}[m]$ and $\mathbf{H}\left[m-M_{\mathrm{d}}\right]$ follow jointly complex Gaussian distribution

$$
\left[\begin{array}{l}
\mathbf{H}_{1}[m] \\
\mathbf{H}_{2}[m] \\
\cdots \\
\mathbf{H}_{K}[m]
\end{array}\right]=\left[\begin{array}{l}
\rho_{\mathrm{d}, 1} \mathbf{H}_{1}\left[m-M_{\mathrm{d}}\right] \\
\rho_{\mathrm{d}, 2} \mathbf{H}_{2}\left[m-M_{\mathrm{d}}\right] \\
\cdots \\
\rho_{\mathrm{d}, K} \mathbf{H}_{K}\left[m-M_{\mathrm{d}}\right]
\end{array}\right]+\left[\begin{array}{l}
\sqrt{1-\left|\rho_{\mathrm{d}, 1}\right|^{2}} \boldsymbol{\varepsilon}_{1}[m] \\
\sqrt{1-\left|\rho_{\mathrm{d}, 2}\right|^{2}} \boldsymbol{\varepsilon}_{2}[m] \\
\cdots \\
\sqrt{1-\left|\rho_{\mathrm{d}, K}\right|^{2}} \boldsymbol{\varepsilon}_{K}[m]
\end{array}\right],
$$

where the elements of the $n_{k} \times M$ random matrix $\varepsilon_{k}$ $[m](k=1,2, \ldots, K)$ are i.i.d zero-mean complex Gaussian random variables with unit variance, $\rho_{\mathrm{d}, k}$ is the complex correlation coefficient between current 
channel gain and the delayed one for user $k$ and is defined as

$$
\rho_{\mathrm{d}, k} \triangleq \mathrm{E}\left\{h_{k, i j}[m] h_{k, i j}^{*}\left[m-M_{\mathrm{d}}\right]\right\}, k=1,2, \ldots, K, \quad i=1,2, \ldots, n_{k,}, \quad j=1,2, \ldots, \mathrm{M},
$$

where $0 \leq\left|\rho_{\mathrm{d}, k}\right| \leq 1$. Because each user can have a different mobile velocity, $\rho_{\mathrm{d}, k}$ of each user can be different.

Defining $\rho_{k} \triangleq \rho_{\mathrm{d}, k} \rho_{\mathrm{d}, k} k=1,2, \ldots, K$, and substituting (5), (7) into (4), the received signal vector (4) of system can be further expressed as

$$
\mathbf{y}[m]=\mathbf{b}_{\text {eq }}[m]+\mathbf{n}_{\text {eq }}[m],
$$

where $\quad \mathbf{b}_{\text {eq }}[m] \triangleq\left[\mathbf{b}_{\text {eq, } 1}^{\mathrm{T}}[m] \mathbf{b}_{\text {eq }, 2}^{\mathrm{T}}[m] \ldots \mathbf{b}_{\text {eq }, K}^{\mathrm{T}}[m]\right]^{\mathrm{T}} \quad$ is referred to as the effective post-processing signal, given by

$$
\mathbf{b}_{\mathrm{eq}}[m]=\frac{1}{\left\|\left(\hat{\mathbf{H}}\left[m-M_{\mathrm{d}}\right]\right)^{\dagger}\right\|_{\mathrm{F}}}\left[\begin{array}{l}
\rho_{1}[m] \mathbf{b}_{1}[m] \\
\rho_{2}[m] \mathbf{b}_{2}[m] \\
\cdots \\
\rho_{K}[m] \mathbf{b}_{K}[m]
\end{array}\right],
$$

while $\quad \mathbf{n}_{\text {eq }}[m] \triangleq\left[\mathbf{n}_{\text {eq, } 1}^{\mathrm{T}}[m] \mathbf{n}_{\text {eq, } 2}^{\mathrm{T}}[m] \ldots \mathbf{n}_{\text {eq, }, K}^{\mathrm{T}}[m]\right]^{\mathrm{T}} \quad$ is referred to as the effective post-processing noise, given by

$$
\mathbf{n}_{\mathrm{eq}}[m]=\left[\begin{array}{l}
\rho_{\mathrm{d}, 1} \sqrt{1-\left|\rho_{\mathrm{e}, 1}\right|^{2}} \boldsymbol{\zeta}_{1}\left[m-M_{\mathrm{d}}\right]+\sqrt{1-\left|\rho_{\mathrm{d}, 1}\right|^{2}} \boldsymbol{\varepsilon}_{1}[m] \\
\rho_{\mathrm{d}, 2} \sqrt{1-\left|\rho_{\mathrm{e}, 2}\right|^{2}} \boldsymbol{\zeta}_{2}\left[m-M_{\mathrm{d}}\right]+\sqrt{1-\left|\rho_{\mathrm{d}, 2}\right|^{2}} \boldsymbol{\varepsilon}_{2}[m] \\
\cdots \\
\rho_{\mathrm{d}, K} \sqrt{1-\left|\rho_{\mathrm{e}, K}\right|^{2}} \boldsymbol{\zeta}_{K}\left[m-M_{\mathrm{d}}\right]+\sqrt{1-\left|\rho_{\mathrm{d}, K}\right|^{2}} \boldsymbol{\varepsilon}_{K}[m]
\end{array}\right] \frac{\left(\hat{\mathrm{H}}\left[m-M_{\mathrm{d}} \mid\right)^{\dagger}\right.}{\|\left(\hat{\mathrm{H}}\left[m-M_{\mathrm{d}} \mid\right)^{\dagger} \|_{\mathrm{F}}\right.} \mathbf{b}[m]+\mathrm{n}[m],
$$

the covariance matrix of $\mathbf{n}_{\text {eq, } k}[m]$ can be computed as

$$
E\left[\mathbf{n}_{\mathrm{eq}, k}[m] \mathbf{n}_{\mathrm{eq}, k}^{\mathrm{H}}[m]\right]=E_{\mathrm{s}}\left(1-\left|\rho_{k}\right|^{2}\right) \mathbf{I}_{n_{k}}+N_{0, k} \mathbf{I}_{n_{k}} .
$$

Because ZF precoding has already separated all data streams at transmitter side, from the receiver's perspective MU-MIMO system has reduced into a lot of parallel "equivalent SISO systems" one of which bears one data stream. Although a more complicated receiver could be used in each "equivalent SISO system" to demodulate the data stream to obtain better performance, the main purpose of this article is to investigate the impact of CSI delay and channel estimation error on MU-MIMO systems, so we use the simple receiver "ZF equalizer" in this article to demodulate each data stream.

Therefore, based on (10) and (12), the post-processing SINR per symbol on the $i$ th stream of user $k$, denoted by $\gamma_{k, i}[m]$, can be obtained as follows

$$
\gamma_{k, i}[m]=\frac{\gamma_{\mathrm{s}, k}\left|\rho_{k}\right|^{2}}{\left[\gamma_{\mathrm{s}, k}\left(1-\left|\rho_{k}\right|^{2}\right)+1\right] \|\left(\hat{\mathrm{H}}\left[m-M_{\mathrm{d}} \mid\right)^{4} \|_{\mathrm{F}}^{2}\right.}, \quad k=1,2, \ldots, K, \quad i=1,2, \ldots, n_{k,}
$$

where $\gamma_{\mathrm{s}, k}=E_{\mathrm{s}} / N_{0, k}$ is the pre-processing SNR of downlink data symbol. Note that all data streams to the same user have the same SINR because we do not consider the power allocation strategy for all data streams and each data stream has the equal power.

Based on (13), we below derive the expression for the average BER of TDD MU-MIMO ZF systems with MQAM modulated signals.

If SNR for uplink pilot symbols is $\gamma_{\mathrm{p}, k}$ and minimum mean-square error (MMSE) is chosen for channel estimation for user $k$, we can deduce

$$
\left|\rho_{\mathrm{e}, k}\right|=\sqrt{\frac{\gamma_{\mathrm{p}, k}}{1+\gamma_{\mathrm{p}, k}}}, \quad k=1,2, \ldots, K
$$

For a time-varying Rayleigh fading channel, its power spectrum follows the Jakes model [20], then

$$
\rho_{\mathrm{d}, k}=J_{0}\left(2 \pi M_{\mathrm{d}} T_{\mathrm{s}} F_{\mathrm{d}, k}\right), \quad k=1,2, \ldots, K,
$$

where $J_{0}$ is a zeroth-order Bessel function of the first kind, $F_{\mathrm{d}, \mathrm{k}}$ is the maximal Doppler frequency shift of user $k, T_{\mathrm{s}}$ is the symbol duration, and $M_{\mathrm{d}} T_{\mathrm{s}}$ is the time delay between uplink channel estimation and downlink data transmission. So,

$$
\rho_{k}=J_{0}\left(2 \pi M_{\mathrm{d}} T_{\mathrm{s}} F_{\mathrm{d}, k}\right) \sqrt{\frac{\gamma_{\mathrm{p}, k}}{1+\gamma_{\mathrm{p}, k}}}, \quad k=1,2, \ldots, K .
$$

If the uncoded M-QAM is used for transmitting signals and the constellation size is $M=2^{q}$, BER in AWGN is [21]

$$
p_{\mathrm{b}} \approx 0.2 \exp \left\{-\frac{1.6 \gamma}{2^{q}-1}\right\},
$$

where $\gamma$ is post-processing SNR.

Substituting (13) into (17), then the BER at the $m$ th symbol interval, denoted by $p_{\mathrm{b}, k, i}[m]$, is as follows for the $i$ th stream of user $k$.

$$
p_{\mathrm{b}, k, i}[m] \approx 0.2 \exp \left\{-\frac{1.6 \gamma_{k, i}[m]}{2^{q}-1}\right\}, \quad k=1,2, \ldots, K, \quad i=1,2, \ldots n_{k} .
$$

It is observed from (13) and (18) that $p_{\mathrm{b}, k, i}[m]$ is dependent on random matrix $\hat{\mathbf{H}}\left[m-M_{\mathrm{d}}\right]$, so we need to calculate the expectation of $p_{\mathrm{b}, k, i}[m]$ with respect to $\hat{\mathbf{H}}\left[m-M_{\mathrm{d}}\right]$ as follows.

\section{Define}

$$
c_{k}^{\prime} \triangleq \frac{\gamma_{\mathrm{s}, k}\left|\rho_{k}\right|^{2}}{\gamma_{\mathrm{s}, k}\left(1-\left|\rho_{k}\right|^{2}\right)+1},
$$

$x \triangleq\left\|\left(\hat{\mathrm{H}}\left[m-M_{\mathrm{d}}\right]\right)^{\dagger}\right\|_{\mathrm{F}}^{2}, \quad$ and $\quad c_{k} \triangleq \frac{1.6 c_{k}^{\prime \prime}}{2^{q}-1}, \quad$ so $p_{b, k, i}[m] \approx 0.2 \exp \left\{-\frac{1.6 c_{k}{ }^{\prime}}{\left(2^{q}-1\right) x}\right\}=0.2 \exp \left\{-\frac{c_{k}}{x}\right\}$. Let the singular-value decomposition of $\hat{\mathbf{H}}$ as follows: 


$$
\hat{\mathbf{H}}=\mathbf{U} \Sigma \mathbf{V}^{\mathrm{H}}
$$

where $\mathbf{U}$ and $\mathbf{V}$ are unitary matrixes and $\Sigma$ is a diagonal matrix of singular values $\left\{\sigma_{i}{ }^{\prime}\right\}$. So, we have

$$
\begin{aligned}
\hat{\mathbf{H}}^{\dagger} & =\hat{\mathbf{H}}^{\mathrm{H}}\left(\hat{\mathbf{H}} \hat{H}^{\mathrm{H}}\right)^{-1}=\left(\mathbf{U} \boldsymbol{\Sigma} \mathbf{V}^{\mathrm{H}}\right)^{\mathrm{H}}\left(\mathbf{U} \boldsymbol{\Sigma} \mathbf{V}^{\mathrm{H}}\left(\mathbf{U} \boldsymbol{\Sigma} \mathbf{V}^{\mathrm{H}}\right)^{\mathrm{H}}\right)^{-1}=\mathbf{V} \boldsymbol{\Sigma}^{\mathrm{H}} \mathbf{U}^{\mathrm{H}}\left(\mathbf{U} \boldsymbol{\Sigma} \mathbf{V}^{\mathrm{H}} \mathbf{V} \boldsymbol{\Sigma}^{\mathrm{H}} \mathbf{U}^{\mathrm{H}}\right)^{-1} \\
& =\mathbf{V} \boldsymbol{\Sigma}^{\mathrm{H}} \mathbf{U}^{\mathrm{H}}\left(\mathbf{U} \boldsymbol{\Sigma} \boldsymbol{\Sigma}^{\mathrm{H}} \mathbf{U}^{\mathrm{H}}\right)^{-1}=\mathbf{V} \boldsymbol{\Sigma}^{\mathrm{H}} \mathbf{U}^{\mathrm{H}}=\left(\boldsymbol{\Sigma} \boldsymbol{\Sigma}^{\mathrm{H}} \mathbf{U} \mathbf{U}^{\mathrm{H}}\right)^{-1}=\mathbf{V} \boldsymbol{\Sigma}^{\mathrm{H}} \mathbf{U}^{\mathrm{H}}\left(\boldsymbol{\Sigma} \boldsymbol{\Sigma}^{\mathrm{H}}\right)^{-1} \\
& =\mathbf{V} \boldsymbol{\Sigma}^{\mathrm{H}}\left(\boldsymbol{\Sigma} \boldsymbol{\Sigma}^{\mathrm{H}}\right)^{-1} \mathbf{U}^{\mathrm{H}}=\mathbf{V} \boldsymbol{\Sigma}^{-1} \mathbf{U}^{\mathrm{H}}
\end{aligned}
$$

which means that the singular value $\lambda_{i}$ of $\hat{\mathbf{H}}^{\dagger}$ is equal to $\frac{1}{\sigma_{i}{ }^{\prime}}$. Then according to matrix knowledge [22], there exists $\left\|(\hat{\mathbf{H}})^{\dagger}\right\|_{\mathrm{F}}^{2}=\sum_{i=1}^{N} \lambda_{i}^{2}$. Therefore, we can obtain

$$
x=\sum_{i=1}^{N} \frac{1}{\left(\sigma_{i}^{\prime}\right)^{2}}=\sum_{i=1}^{N} \frac{1}{\sigma_{i}}
$$

where $\sigma_{i}=\left(\sigma_{i}^{\prime}\right)^{2}$. So, $p_{\mathrm{b}, k, i}$ now depends on the square $\sigma_{i}$ of each singular value $\sigma_{i}{ }^{\prime}$ of $\hat{\mathbf{H}}\left[m-M_{\mathrm{d}}\right]$.

As mentioned previously, the entries of $\hat{\mathbf{H}}\left[m-M_{\mathrm{d}}\right]$ are i.i.d zero-mean complex Gaussian random variables with unit variance, so the joint probability density function (PDF) of the square $\sigma_{i}$ of all singular values $\sigma_{i}{ }^{\prime}$ of $\hat{\mathbf{H}}\left[m-M_{\mathrm{d}}\right]$, denoted by $f\left(\sigma_{1}, \sigma_{2}, \ldots, \sigma_{N}\right)$, can be written as follows according to Theorem 2.17 of [23]

$$
f\left(\sigma_{1}, \sigma_{2}, \ldots, \sigma_{N}\right)=e^{-\sum_{i=1}^{N} \sigma_{i}} \prod_{i=1}^{N} \frac{\sigma_{i}^{M-N}}{(N-i) !(M-i) !} \prod_{i<j}^{N}\left(\sigma_{i}-\sigma_{j}\right)^{2} .
$$

Hence, the average BER, denoted by $\tilde{p}_{\mathrm{b}, k, i}$, can be obtained as follows

$$
\tilde{p}_{\mathrm{b}, k, i}\left(c_{k}, N, M\right) \approx \int_{0}^{+\infty} \int_{0}^{\sigma_{1}} \cdots \int_{0}^{\sigma_{N-1}} 0.2 \exp \left(-c_{k} / \sum_{i=1}^{N} \frac{1}{\sigma_{i}}\right) f\left(\sigma_{1}, \sigma_{2}, \ldots, \sigma_{N}\right) d \sigma_{N} \ldots d \sigma_{2} d \sigma_{1} .
$$

While it is difficult to obtain a closed-form expression for (22), the integral is fairly straightforward to evaluate numerically, at least when $\min (M, N)$ is small (in practical communication systems, the number of antennas of $\mathrm{BS}$ is at most eight at present), so it is valuable for the design of the adaptive modulation and coding scheme in practical communication systems. Moreover, since the BER expression includes the parameters related to channel conditions (e.g., Doppler frequency shift, uplink pilot SNR, CSI delay length, etc.) and the parameters related to system configurations (e.g., modulation mode, symbol duration, number of BS antennas, number of UE antenna, etc.), it provides the hints for people to optimize the MU-MIMO performance in TDD systems from different perspectives.
The BER function $\tilde{p}_{\mathrm{b}, k, i}$ is only determined by three parameters including $c_{k}, N$, and $M$. Hence, we can summarize the impact of imperfect CSI as follows.

\section{Increase BER}

As $M_{\mathrm{d}} T_{s} F_{\mathrm{d}, k}$ increases or $\gamma_{\mathrm{p}, k}$ decreases, $\left|\rho_{k}\right|$ decreases, so $c_{k}$ decreases and $\tilde{p}_{\mathrm{b}, k, i}$ in turn increases. In other words, system performance degrades when the Doppler shift is high, or when the SNR of pilot symbols is low.

\section{Error floor}

If CSI is perfect and $\gamma_{\mathrm{s}, k} \rightarrow \infty$, then $c_{k} \rightarrow \infty$ and $\tilde{p}_{\mathrm{b}, k, i} \rightarrow 0$. However, if CSI is imperfect and $\gamma_{\mathrm{s}, k} \rightarrow \infty$,

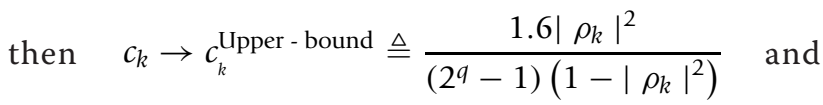
$\tilde{p}_{\mathrm{b}, k, i} \rightarrow \int_{0}^{+\infty} \int_{0}^{\sigma_{1}} \cdots \int_{0}^{\sigma_{N-1}} 0.2 \exp \left(-\frac{-c_{k}^{\text {Upper - bound }}}{\sum_{i=1}^{N} \frac{1}{\sigma_{i}}}\right) f\left(\sigma_{1}, \sigma_{2}, \ldots, \sigma_{N}\right) d \sigma_{N} \ldots d \sigma_{2} d \sigma_{1}$, which means $c_{k}$ approaches an upper-bound and the BER thus exhibits an error floor when $\gamma_{\mathrm{s}, k}$ is high, further increases in $\gamma_{\mathrm{s}, k}$ gain nothing. This error floor worsens as $\left|\rho_{k}\right|$ decreases, i.e., as the channel estimation error or CSI delay of user $k$ increases.

\section{Simulation results}

Consider an LTE TDD downlink MU-MIMO system where a BS with eight antennas transmits data to two users each equipped with two antennas. The channels are assumed to be time-varying, spatially uncorrelated, frequency flat, and Rayleigh fading. Jakes model is used to simulate the time-varying channels. The carrier frequency is $2.3 \mathrm{GHz}$. Symbol interval is $1 / 14 \mathrm{~ms}$. TDD frame configuration 2 is used to transmit downlink data block and uplink pilots for downlink beamforming transmission are sent in the last symbol of the uplink subframe. As shown in Figure 1, the ratio of uplink subframes to downlink subframes is 1:3 in one equivalent block where the first $1 \mathrm{~ms}$ is for uplink and other $3 \mathrm{~ms}$ is for downlink, so the range of CSI delay $M_{\mathrm{d}}$ is from 1 to 42 symbol intervals for the different downlink data symbol. For simplification, we make the following assumptions: (1) The noise covariance $N_{0, k}$ of each user is the same, which implies $\gamma_{\mathrm{s}, k}$ of each user is the same, (2) uplink pilot SNR $\gamma_{\mathrm{p}, k}$ of each user is the same, and is equal to the pre-processing SNR $\gamma_{\mathrm{s}, k}$ of downlink data symbol when the channel estimation error of CSI is considered, (3) no channel coding is considered. Owing to the assumptions above, we can ignore user index $k$ for all related variables hereafter, e.g., replacing $\gamma_{\mathrm{s}, k}$ with $\gamma_{\mathrm{s}}$. MMSE channel estimation and ideal channel 
estimation are used by BS and each user, respectively. Simulation parameters, some of which are cited from $[18,19]$, are summarized in Table 1.

According to the system configuration in Table 1, the average BER $\tilde{p}_{\mathrm{b}, k, i}$ in (22) becomes into

$$
\tilde{p}_{b, k, i}\left(c_{k,}, 4,8\right) \approx \int_{0}^{+\infty} \int_{0}^{\sigma_{1}} \cdots \int_{0}^{\sigma_{3}} 0.2 \exp \left(-c_{k} / \sum_{i=1}^{4} \frac{1}{\sigma_{i}}\right) f\left(\sigma_{1}, \sigma_{2}, \ldots, \sigma_{4}\right) d \sigma_{4} \ldots d \sigma_{2} d \sigma_{1} .
$$

where

$$
f\left(\sigma_{1}, \sigma_{2}, \ldots, \sigma_{4}\right)=e^{-\sum_{i=1}^{4} \sigma_{i}} \prod_{i=1}^{N} \frac{\sigma_{i}^{4}}{(4-i) !(8-i) !} \prod_{i<j}^{4}\left(\sigma_{i}-\sigma_{j}\right)^{2} .
$$

The above integral can be evaluated with numerical calculation software, e.g., Matlab (2009a), Mathematica, etc.

Figures 3, 4, and 5 show the variation of system BER (averaged over the two users) with $\gamma_{\mathrm{s}}$ for 4QAM, 16QAM, and 64QAM, respectively. In each figure, the four typical cases are considered according to the four kinds of different relationships between CSI and downlink transmission channel:

1. Without CSI delay and without estimation error: 0 $\mathrm{km} / \mathrm{h}$ and $\gamma_{\mathrm{p}}=\infty$

There are no CSI delay and no estimation error between CSI and downlink transmission channel. It is the perfect CSI case which is as the comparison baseline for other three cases.

2. Without CSI delay and with estimation error: $0 \mathrm{~km} /$ $\mathrm{h}$ and $\gamma_{\mathrm{p}}=\gamma_{\mathrm{s}}$

There is no CSI delay but exists estimation error between CSI and downlink transmission channel.

3. With CSI delay and without estimation error: 10 $\mathrm{km} / \mathrm{h}$ and $\gamma_{\mathrm{p}}=\infty$

There exists CSI delay but is no estimation error between CSI and downlink transmission channel.

4. With CSI delay and with estimation error: $10 \mathrm{~km} / \mathrm{h}$ and $\gamma_{\mathrm{p}}=\gamma_{\mathrm{s}}$
There are both CSI delay and estimation error between CSI and downlink transmission channel.

One can see that the simulation curves match the analytical ones very well, demonstrating the correctness of our average BER expression. It is also observed that, the BER increases as the channel estimation error and/or CSI delay (or mobile velocity) increase(s), and an error floor is evident at high SNR for the cases with CSI delay, which agrees with our summary about imperfect CSI impact. Furthermore, one can find that channel estimation error causes array gain loss by comparing the curves of the same mobile velocity but with different pilot SNR while CSI delay causes diversity gain loss by comparing the curves of the same pilot SNR but with different mobile velocities. Moreover, CSI delay causes more performance degradation at high SNR than channel estimation error as the latter diminishes when the SNR is high.

Figure 6 illustrates the variation of system BER with delay $M_{\mathrm{d}}$ in the case of 4QAM, 16QAM, and 64QAM. Here, $\gamma_{s}$ and $\gamma_{p}$ are fixed as $30 \mathrm{~dB}$ in order to ignore the impact of channel estimation error as much as possible, the range of $M_{\mathrm{d}}$ comes from linear area $0 \leq x \leq 2$ of $J_{0}$ $(x), F_{\mathrm{d}}$ is $5 \mathrm{~Hz}$. One can see that BER shows the trend of increasing as $M_{\mathrm{d}}$ increases and finally reaching error floor. The reason is that CSI becomes more and more imperfect as $M_{\mathrm{d}}$ increases, so BER becomes more and more big; when $M_{\mathrm{d}}$ is beyond a certain long delay value, CSI has already become saturated imperfect, so BER arrives at error floor.

Figure 7 illustrates the variation of system BER with $\gamma_{p}$ (reflecting channel estimation error) in the case of 4QAM, 16QAM, and 64QAM. Here, $\gamma_{\mathrm{s}}$ are fixed as 10 $\mathrm{dB}$, and $M_{\mathrm{d}}$ is fixed as 10 symbol intervals in order to ignore the impact of CSI delay as much as possible, $F_{\mathrm{d}}$ is $5 \mathrm{~Hz}$. One can see that BER decreases as $\gamma_{\mathrm{p}}$ increases and finally arrives at error floor. The reason is that CSI become more and more perfect as $\gamma_{\mathrm{p}}$ increases; so BER

Table 1 Simulation parameters

\begin{tabular}{lll}
\hline Parameter & value & Comment \\
\hline$T_{\mathrm{s}}($ second) & $10^{-3} / 14$ & Symbol interval \\
$f_{\mathrm{C}}(\mathrm{GHz})$ & 2.3 & Carrier frequency \\
$M_{\mathrm{d}}$ (in symbol) & $1,2, \ldots, 42$ & CSI delay in symbol for different downlink data symbol \\
$n_{k}$ & 2 & Number of antennas of each user \\
$M$ & 8 & Number of antennas of BS \\
$K$ & 2 & Number of user \\
$N$ & 4 & Number of antennas of all users \\
$\gamma_{\mathrm{s}}(\mathrm{dB})$ & $0-30$ & Pre-processing data SNR \\
$\gamma_{\mathrm{p}}(\mathrm{dB})$ & $0-30$ & Pre-processing pilot SNR \\
$v(\mathrm{~km} / \mathrm{h})$ & 0,10 & Mobile velocity of user \\
Modulation & $4,16,64$ & 4QAM, 16QAM and 64QAM are used respectively for modulation \\
\hline
\end{tabular}




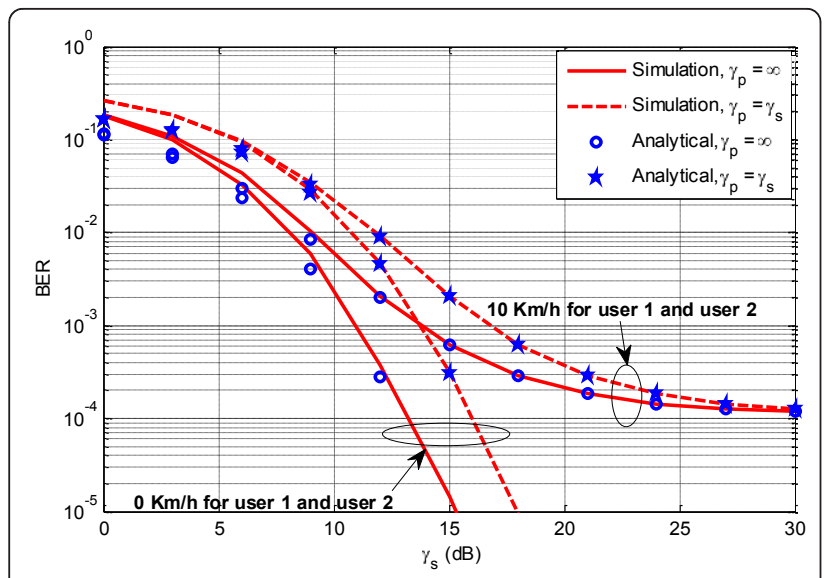

Figure 3 BER performance of MU-MIMO downlink system (4QAM).

becomes more and more small; when $\gamma_{\mathrm{p}}$ becomes high, channel estimation error diminishes and CSI delay dominates BER, so BER arrives at an error floor, which again agrees with our summary about imperfect CSI impact.

Figure 8 depicts the variation of system BER with the speed of user in the case of 4QAM, 16QAM, and 64QAM. Here, $\gamma_{\mathrm{s}}$ and $\gamma_{\mathrm{p}}$ are fixed as $30 \mathrm{~dB}$ in order to ignore the impact of channel estimation error as much as possible, $M_{\mathrm{d}}$ is fixed as 10 symbol intervals, the speeds of both users are assumed as the same for simplification and change from 0 to $100 \mathrm{~km} / \mathrm{h}$. One can see that BER increases as the speed of user increases and finally reaches error floor. The reason is that Doppler frequency shift becomes more and more big as the speed of user increases, which causes that CSI becomes more and more imperfect, so BER becomes more and more big. When the speed of user is beyond a certain value, CSI has already become saturated imperfect, so BER arrives at error floor. Moreover, by comparing

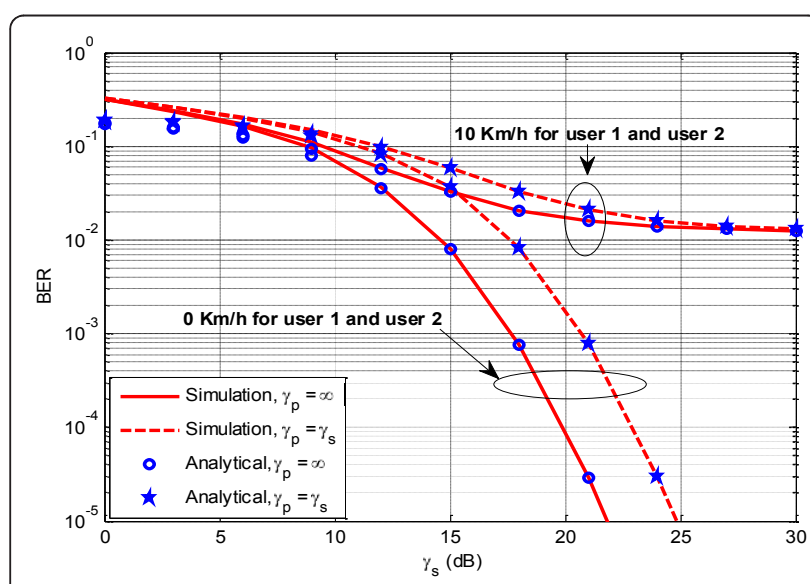

Figure 4 BER performance of MU-MIMO downlink system (16QAM).

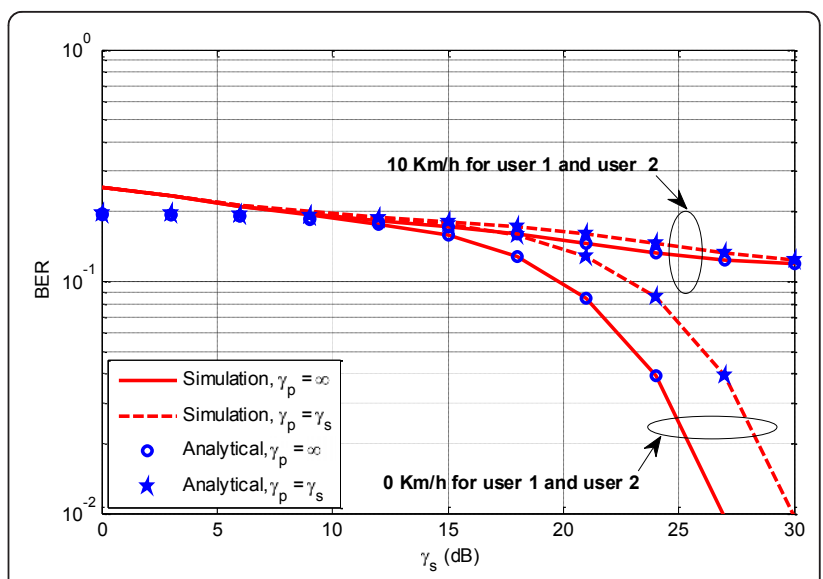

Figure 5 BER performance of MU-MIMO downlink system (64QAM)

Figures 6 and 8 , we can find that the impact of the speed of user on BER is similar to the impact of CSI delay $M_{\mathrm{d}}$ on BER and the big speed value is equivalent to the big CSI delay value, which can be explained by (15). It should be pointed out that in practical communications systems, the CSI delay $M_{\mathrm{d}}$ is generally fixed because of the selected frame structure in advance while the speed of user often changes.

Figure 9 depicts the variation of system BER with the number of user in the case of 4QAM, 16QAM, and 64QAM. Here, $\gamma_{s}$ and $\gamma_{p}$ are fixed as $30 \mathrm{~dB}, M_{\mathrm{d}}$ is fixed as 10 symbol intervals. For simplification, we make the following assumptions: (1) each user is equipped with one antenna, so the number of user $K$ is equal to the total number of antennas of all users $N$; (2) the speed of each user is the same and corresponding Doppler frequency shift is $5 \mathrm{~Hz}$; (3) the number of user changes from 1 to 8 because of the condition $M=8 \geq K=N=\sum_{k=1}^{K} n_{k}$. One can see that BER

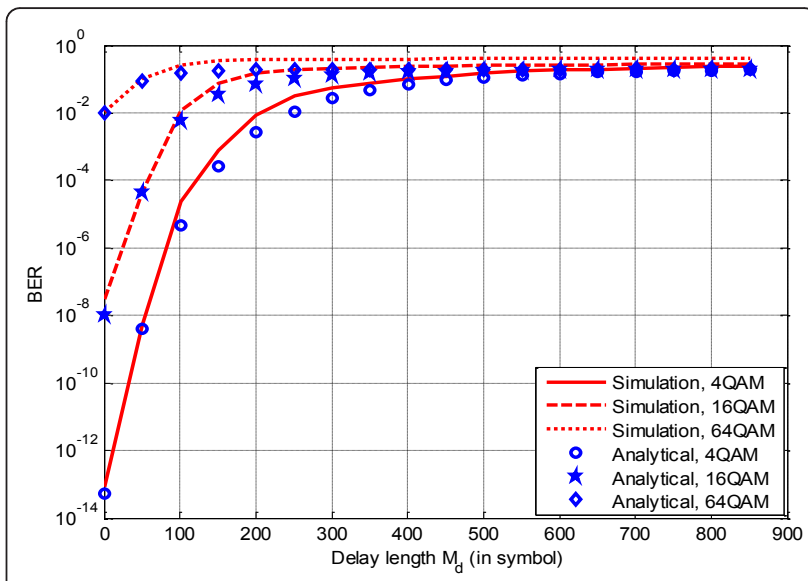

Figure 6 Relationship between BER and delay $M_{\mathrm{d}}$ 


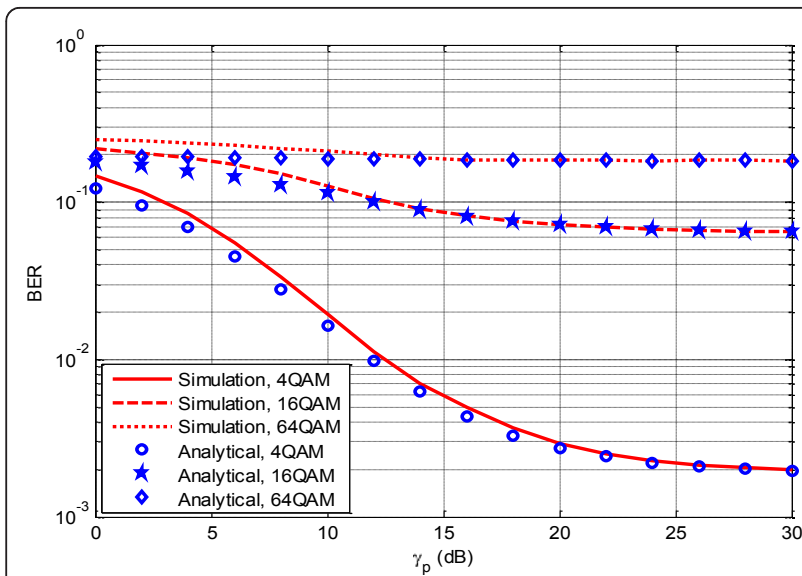

Figure 7 Relationship between BER and $\gamma_{p}$

increases as the number of user increases. The reason is that inter-user interferences inevitably exist because of the existences of estimation error and CSI delay between CSI and downlink transmission channel, and increase as the number of user increases, so the BER becomes more and more big.

\section{Conclusion}

In this article, we have investigated the BER of TDD downlink MU-MIMO ZF systems in the presence of imperfect CSI. By exploiting the correlation between the actual channel and the estimated one as well as channel time-correlation, we have developed the novel BER expression for TDD downlink MU-MIMO systems with M-QAM-modulated signals. Furthermore, we find that CSI delay and channel estimation error degrade system performance and even cause error floor, among which channel estimation error causes array gain loss while CSI delay causes diversity gain loss. At high SNR, CSI

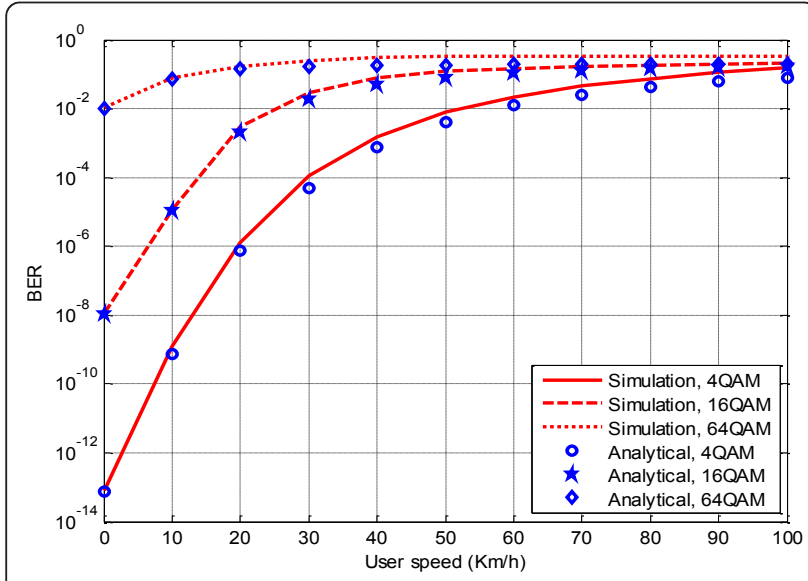

Figure 8 Relationship between BER and user speed.

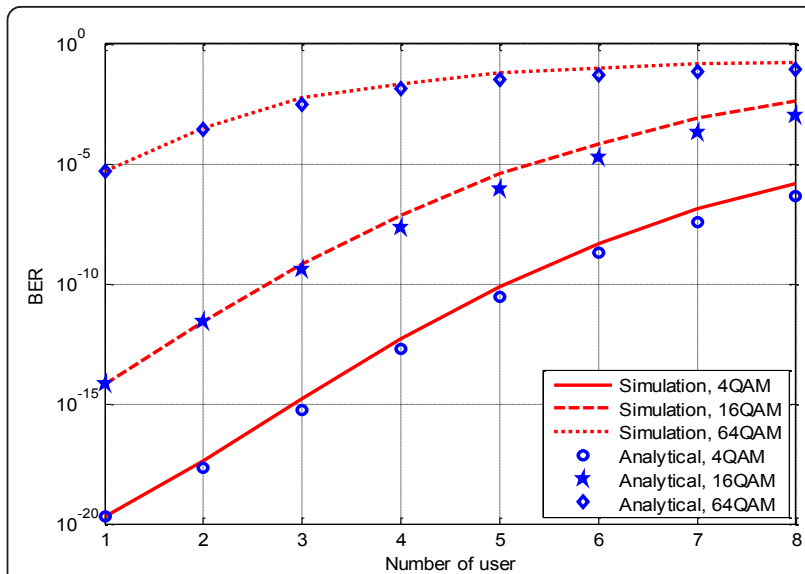

Figure 9 Relationship between BER and the number of user

delay causes more performance degradation than channel estimation error. Especially, our research is valuable for the design of the adaptive modulation and coding scheme as well as the optimization of MU-MIMO systems. Numerical simulations have verified our theoretical analysis.

\section{Acknowledgements}

This paper was supported jointly by China Middle\&Long term project "Next generation wideband wireless communications network"(2010ZX03002-003), National Nature Science Foundation of China (No. 60872017, No. 60832009), important National Science \& Technology Specific projects (No. 2010ZX03003-002-03, No. 2011ZX03003-001-03), and Chinese National Programs for high technology research development project (No.2009AA011505), and important National Science \& Technology Specific Projects (No. 2011ZX03003-001-03)

\section{Author details}

'Department of Electronic Engineering, Shanghai Jiao Tong University, Shanghai, P.R. China ${ }^{2}$ Wireless R\&D, Alcatel-Lucent Shanghai Bell, Shanghai, P.R. China

\section{Competing interests}

The authors declare that they have no competing interests.

Received: 23 March 2011 Accepted: 16 November 2011 Published: 16 November 2011

\section{References}

1. T Koivisto, LTE-advanced research in 3GPP, in Nokia, GIGA Seminar'08 (4 Dec2008)

2. D Gesbert, M Kountouris, RW Heath Jr, C Chae, T Sälzer, Shift the memo paradigm: from single-user to multiuser communications. IEEE Signal Process Mag. 24(5), 36-46 (2007)

3. M Costa, Writing on dirty paper. IEEE Trans Inf Theory. 29(3), 439-441 (1983). doi:10.1109/TIT.1983.1056659

4. LU Choi, RD Murch, A transmit preprocessing technique for multiuser mimo systems using a decomposition approach. IEEE Trans Wirel Commun. 3(1), 20-24 (2004). doi:10.1109/TWC.2003.821148

5. QH Spencer, AL Swindlehurst, M Haardt, Zero-forcing methods for downlink spatial multiplexing in multiuser mimo channels. IEEE Trans Signal Process. 52(2), 461-471 (2004). doi:10.1109/TSP.2003.821107

6. CB Peel, BM Hochwald, AL Swindlehurst, A vector perturbation technique for near-capacity multiantenna multiuser communication-part I: channel 
inversion and regularization. IEEE Trans Commun. 53(1), 195-202 (2005). doi:10.1109/TCOMM.2004.840638

7. G Caire, N Jindal, M Kobayashi, N Ravindran, Multiuser MIMO achievable rates with downlink training and channel state feedback. IEEE Trans Inf Theory. 56(6), 2845-2866 (2010)

8. L Musavian, S Aissa, On the achievable sum-rate of correlated mimo multiple access channel with imperfect channel estimation. IEEE Trans Wirel Commun. 7(7), 2549-2559 (2008)

9. J Zhang, JG Andrews, RW Heath Jr, Single-user MIMO vs. multiuser MIMO in the broadcast channel with CSIT constraint. in 46th Annual Allerton Conference on Communication, Control, and Computing, Allerton House, (September 24-26, 2008)

10. J Zhang, M Kountouris, JG Andrews, RW Heath Jr, Multi-mode transmission for the MIMO broadcast channel with imperfect channel state information IEEE Trans Commun. 59(3), 803-814 (2011)

11. C Wang, RD Murch, On the sum-rate scaling law of downlink MU-MIMO decomposition transmission with fixed quality imperfect CSIT. IEEE Trans Wirel Commun. 8(1), 113-117 (2009)

12. B Song, M Haardt, Effects of imperfect channel state information on achievable rates of precoded multi-user MIMO broadcast channels with limited feedback. in IEEE International Conference on Communications (ICC 2009), Dresden Germany, (14-18 June 2009)

13. R Annavajjala, PC Cosman, LB Milstein, Performance analysis of linear modulation schemes with generalized diversity combining on rayleigh fading channels with noisy channel estimates. IEEE Trans Inf Theory. 53(12), 4701-4727 (2007)

14. EN Onggosanusi, A Gatherer, AG Dabak, Performance analysis of closedloop transmit diversity in the presence of feedback delay. IEEE Trans Commun. 49(9), 1618-1630 (2001). doi:10.1109/26.950348

15. J Mundarath, P Ramanathan, B Van Veen, A distributed downlink scheduling method for multi-user communication with zero-forcing beamforming. IEEE Tran Wirel Commun. 7(11), 4508-4521 (2008)

16. S Sigdel, RC Elliott, WA Krzymien, M Al-Shalash, Greedy and genetic user scheduling algorithms for multiuser MIMO systems with block diagonalization. in IEEE Vehicular Technology Conference Fall (VTC 2009-Fall). Anchorage, Alaska, USA, (September 20-23, 2009)1-6

17. TS Rappaport, Wireless Communication Principles and Practice, (New York: Prentice Hall, 1998)

18. 3GPP, 3GPP TSG RAN: TS36.212v8.9.0 E-UTRA. Physical Channels and Modulation (December 2009)

19. 3GPP, 3GPP TSG RAN: TS36.212v9.1.0 E-UTRA. Physical Channels and Modulation (March 2010)

20. WC Jakes, Microwave Mobile Communications, (IEEE Press, 1993)

21. ST Chung, AJ Goldsmith, Degrees of freedom in adaptive modulation: a unified view. IEEE Trans Commun. 49(9), 1561-1571 (2001). doi:10.1109/ 26.950343

22. XD Zhang, Matrix Analysis and Applications, (Tsinghua University Press, 2004)

23. AM Tulino, S Verdu, Random Matrix Theory and Wireless Communications, (Now Publisher Inc, 2004)

doi:10.1186/1687-6180-2011-104

Cite this article as: Zhou et al:: BER analysis of TDD downlink multiuser MIMO systems with imperfect channel state information. EURASIP

Journal on Advances in Signal Processing 2011 2011:104.

\section{Submit your manuscript to a SpringerOpen ${ }^{\mathcal{O}}$ journal and benefit from:}

- Convenient online submission

- Rigorous peer review

- Immediate publication on acceptance

- Open access: articles freely available online

- High visibility within the field

- Retaining the copyright to your article

Submit your next manuscript at $\gg$ springeropen.com 